\title{
Refractive Outcomes Using the Lenstar Optical Low Coherence Reflectometry Biometer in Phacovitrectomy for Epiretrinal Membranes and Macular Holes
}

\author{
Mark A. P. Fajgenbaum, Julian Robins, Tom H. Williamson \\ Ophthalmology Department, St. Thomas' Hospital, London, UK \\ Email: markfajgenbaum@gmail.com
}

How to cite this paper: Fajgenbaum, M.A.P., Robins, J. and Williamson, T.H. (2017) Refractive Outcomes Using the Lenstar Optical Low Coherence Reflectometry Biometer in Phacovitrectomy for Epiretrinal Membranes and Macular Holes. Open Journal of Ophthalmology, 7, 216-224.

https://doi.org/10.4236/ojoph.2017.73029

Received: July 20, 2017

Accepted: August 15, 2017

Published: August 18, 2017

Copyright $\odot 2017$ by authors and Scientific Research Publishing Inc. This work is licensed under the Creative Commons Attribution International License (CC BY 4.0).

http://creativecommons.org/licenses/by/4.0/

\section{Open Access}

\begin{abstract}
Objective: To compare refractive outcomes using the Lenstar optical low-coherence reflectometry (OLCR) biometer in the following cases: phacovitrectomy for epiretinal membranes and macular holes (phacovitrectomy group); sequential cataract surgery after prior vitrectomy (sequential-phaco group); routine cataract surgery (phaco-only group). Methods: This study was a retrospective, consecutive comparative series. Main outcomes were mean prediction error (ME) and mean absolute prediction error (MAE). Secondary outcome was the variance in prediction error. Results: ME was significantly more myopic in the phacovitrectomy group $(-0.08 \pm 0.77 \mathrm{D}$, mean \pm $\mathrm{SD}, \mathrm{p}=0.04)$ and the sequential-phaco group $(-0.09 \pm 0.51 \mathrm{D}, \mathrm{p}=0.01)$ compared to the phaco-only group $(+0.24 \pm 0.53 \mathrm{D})$. MAEs were not statistically different across the 3 groups. The phacovitrectomy group had a wider variance in prediction errors compared to the phaco-only group $(\mathrm{p}=0.03)$. Conclusions: There is a myopic shift of approximately $-0.3 \mathrm{D}$ in both phacovitrectomy and sequential-phaco cases compared to phaco-only cases when using OLCR-based optical biometry. Phacovitrectomy outcomes are more variable compared to phaco-only eyes.
\end{abstract}

\section{Keywords}

Refractive Outcomes, Phacovitrectomy, Optical Biometry, Lenstar, OLCR

\section{Introduction}

Vitrectomy is commonly combined with cataract surgery (phacovitrectomy) in the management of phakic eyes with macular holes $(\mathrm{MH})$ or epiretinal membranes (ERM) [1]. Regardless of the lens status, phacovitrectomy may be preferred to vi- 
trectomy alone in order to pre-empt the progression of post-vitrectomy cataract and provide quicker overall visual recovery [2].

While phacovitrectomy is more time- and resource-efficient than sequential cataract surgery, it may be associated with less predictable refractive outcomes. Several groups have reported a myopic shift after phacovitrectomy for macula holes and ERM's, particularly with ultrasound biometry [3]-[8]. Optical biometry is now the gold-standard in pre-operative cataract assessment and may be less prone to myopic shift in phacovitrectomy [9], but there is conflicting evidence [10] [11] and the published literature remains limited. Existing studies on optical biometry in phacovitrectomy have exclusively used the IOLMaster machine (Carl Zeiss Meditec AG, Jena, Germany), which works by partial coherence interferometry (PCI) [9] [10] [11]. We are not aware of refractive outcome data for phacovitrectomy in macular holes and ERM's using newer-generation optical biometry devices based on optical low coherence reflectometry (OLCR).

The aim of our study was to report the accuracy of OLCR biometry obtained from the Lenstar LS900 (Haag-Streit AG, Koniz, Switzerland) in predicting postoperative refraction in eyes undergoing phacovitrectomy for macular pathology (ERM or MH). Secondly, the study aimed to compare outcomes in phacovitrectomy against sequential cases, where phacoemulsification occurred post-vitrectomy, and against routine phacoemulsification surgery in eyes with no prior vitreoretinal history.

\section{Materials and Methods}

This study was approved by our institution's audit committee and adhered to the tenets of the declaration of Helsinki. Refractive outcomes on a prospectively maintained electronic medical record (VITREOR, Axsys Technologies, Glasgow, UK) were reviewed for a consecutive series of patients who had combined vitrectomy and cataract surgery (phacovitrectomy) for either macular hole or ERM performed by a single surgeon (THW) at a single centre over a period of 3 years. Patients were included in the study if they had:

- Optical biometry pre-operatively with the Lenstar LS 900;

- Uneventful phacovitrectomy surgery;

- Implantation of a foldable acrylic intraocular lens (IOL) either SA60AT or SN6CWS (Alcon, Fort Worth, Texas) in the capsular bag.

Only one eye of a patient was allowed inclusion. Where both eyes were eligible the first eye was enrolled in the study. The SRK/T formula was used to calculate IOL power. Optimized A constants were used for each IOL from pooled surgical data available on a regularly updated online database (accessed on Dec. 2016 from URL http://www.ocusoft.de/ulib). Objective post-operative refraction was performed using an autorefractor between 2 and 4 months after surgery, after complete resorption of any intra-vitreal gas tamponade.

In phacovitrectomy cases, phacoemulsification was performed via a $2.75 \mathrm{~mm}$ superior clear corneal incision. IOL insertion took place prior to vitrectomy without wound enlargement using a cartridge introduction system. The incision 
was sutureless. Vitrectomy was performed using the Constellation 25-guage or 23-guage systems (Alcon, Fort Worth, Texas). Sclerostomies were made using an oblique 2-step entry $3.5 \mathrm{~mm}$ from the limbus. When necessary sclerostomies were closed with 9-O polyglactin (Vicryl) sutures to ensure a watertight wound. Gas tamponade was used as necessary. All patients with macular hole had gas tamponade with $\mathrm{C}_{2} \mathrm{~F}_{6}$ and were asked to posture upright by day and not supine at night for 7 days.

Two control groups were utilized. The sequential-phaco control group comprised a consecutive series of eyes that had sequential, uncomplicated phacoemulsification after previously successful vitrectomy for a variety of indications, primarily retinal detachment. The phaco-only control group comprised a consecutive series of eyes that had uneventful phacoemulsification with no previous history of intra-ocular surgery and no known vitreoretinal pathology. In both control groups, phacoemulsification was performed identically to the phacovitrectomy group, by the same surgeon (THW) using a superior, sutureless, 2.75 $\mathrm{mm}$ clear corneal incision. In both groups, optical biometry was obtained with the Lenstar machine pre-operatively and post-operative refraction was determined with an autorefractor, as per the phacovitrectomy group.

Statistical analysis was performed using SPSS version 20 (IBM, Armonk, NY). The difference in spherical equivalent values between actual and target refraction was used to calculate mean post-operative prediction error (ME). The absolute value of the difference in spherical equivalent between actual and target refraction was used to calculate the mean absolute post-operative prediction prediction error (MAE). ME and MAE across groups were compared using non-paired Student $t$ tests after confirming normality of distributions with the Shapiro Wilk test. The variances in prediction error were compared between groups with Levene's test. The proportion of eyes within \pm 0.25 dioptres (D), $\pm 0.50 \mathrm{D}, \pm 1.00 \mathrm{D}$ and $\pm 2.00 \mathrm{D}$ of target refraction was calculated. Proportions were compared between groups using either Chi-squared tests or Fisher's exact test on the basis of expected frequencies.

\section{Results}

There were 36 eyes in the phacovitrectomy group (18 ERM, $18 \mathrm{MH}$ ), 41 eyes in the phaco-only control group and 26 eyes in the sequential-phaco control group. The demographics of the 3 groups are shown in Table 1. The SA60AT lens was used more frequently than the SN6CWS but there was no significant variation in lens usage between groups $(\mathrm{p}=0.92)$. Table 2 shows the refractive outcomes. Both the phacovitrectomy group (ME of $-0.08 \mathrm{D}$ ) and the sequential-phaco group (ME -0.09D) had statistically more myopic mean predictive errors (ME) compared to the phaco-only group (ME $0.24 \mathrm{D})[\mathrm{p}=0.04$ and $\mathrm{p}=0.01$ respectively]. Within the phacovitrectomy group, multivariate regression found the predictive error was not influenced by: macula pathology (ERM vs. MH), IOL type, axial length or the presence of gas tamponade ( $\mathrm{p} \geq 0.1)$. There was no statistical difference in mean absolute predictive error (MAE) across the 3 groups. 
Table 1. Pre-operative demographics.

\begin{tabular}{|c|c|c|c|c|c|c|}
\hline \multirow{2}{*}{ Parameter } & \multicolumn{3}{|c|}{ Phacovitrectomy group } & \multirow{2}{*}{$\begin{array}{c}\text { Sequential-Phaco } \\
\text { group }\end{array}$} & \multirow{2}{*}{$\begin{array}{l}\text { Phaco-only } \\
\text { grouap }\end{array}$} & \multirow{2}{*}{$\mathrm{P}$-value ${ }^{\mathrm{a}}$} \\
\hline & $E R M$ & $M H$ & All cases & & & \\
\hline $\mathrm{N}$ & 18 & 18 & 36 & 26 & 41 & - \\
\hline $\begin{array}{l}\text { Indication (number of } \\
\text { cases) }\end{array}$ & ERM: 18 & MH: 18 & $\begin{array}{l}\text { ERM: } 18 \\
\text { MH: } 18\end{array}$ & $\begin{array}{c}\text { RD: } 9 \\
\text { ERM: } 7 \\
\text { VH: } 2 \\
\text { MH: } 1 \\
\text { Other: } 7\end{array}$ & Cataract: 41 & - \\
\hline Age in years $($ mean $\pm S D)$ & $66 \pm 8$ & $67 \pm 6$ & $66 \pm 7$ & $66 \pm 11$ & $71 \pm 11$ & 0.13 \\
\hline IOL: SA60AT & 11 & 9 & 20 & 14 & 21 & 0.92 \\
\hline SN6WS & 7 & 9 & 16 & 12 & 20 & 0.92 \\
\hline $\begin{array}{c}\text { Gauge of PPV: } 23 \\
25\end{array}$ & $\begin{array}{c}15 \\
3\end{array}$ & $\begin{array}{c}16 \\
2\end{array}$ & $\begin{array}{c}31 \\
5\end{array}$ & $\mathrm{Na}$ & $\mathrm{Na}$ & - \\
\hline Gas Tamponade & $5 / 18^{\mathrm{b}}(28 \%)$ & $18 / 18(100 \%)$ & $23 / 36(64 \%)$ & $\mathrm{Na}$ & $\mathrm{Na}$ & - \\
\hline
\end{tabular}

$\mathrm{ERM}=$ epiretinal membrane; $\mathrm{IOL}$ = intraocular lens; $\mathrm{MH}$ = macula hole; $\mathrm{PPV}=$ pars plana vitrectomy; $\mathrm{RD}$ = retinal detachment; $\mathrm{SD}=$ standard deviation; $\mathrm{VH}=$ vitreous haemorrhage. a. P-value across the 3 groups: phacovitrectomy versus sequential-phaco versus phaco-only; b. 3 cases used $20 \%$ C2F6 and 2 cases used $30 \%$ SF6.

Table 2. Refractive aims, refractive errors, axial lengths and IOL power.

\begin{tabular}{|c|c|c|c|c|c|c|}
\hline \multirow[b]{2}{*}{ Group } & \multicolumn{6}{|c|}{ Mean \pm SD (range) } \\
\hline & $\begin{array}{l}\text { Eyes } \\
\text { (n) }\end{array}$ & $\begin{array}{l}\text { Refractive } \\
\text { aim (D) }\end{array}$ & Prediction error (ME) (D) & Absolute prediction error (MAE) (D) & $\begin{array}{c}\mathrm{AL} \\
(\mathrm{mm})\end{array}$ & $\begin{array}{l}\text { IOL power } \\
\text { (D) }\end{array}$ \\
\hline \multicolumn{7}{|l|}{ Phacovitrectomy } \\
\hline Macular hole & 18 & $-0.48 \pm 1.00$ & $-0.24 \pm 0.76(-1.48$ to +1.19$)$ & $0.65 \pm 0.44(0.10$ to 1.48$)$ & $24.00 \pm 1.64$ & $20.44 \pm 3.68$ \\
\hline Macular Pucker & 18 & $-0.59 \pm 0.98$ & $0.08 \pm 0.77(-2.38$ to +1.05$)$ & $0.50 \pm 0.57(0.01$ to 2.38$)$ & $25.13 \pm 1.52$ & $17.44 \pm 4.42$ \\
\hline All cases & 36 & $-0.54 \pm 0.98$ & $-0.08 \pm 0.77(-2.38$ to +1.19$)$ & $0.58 \pm 0.51(0.01$ to 2.38$)$ & $24.56 \pm 1.66$ & $18.94 \pm 4.29$ \\
\hline Phaco-only & 41 & $-0.60 \pm 0.57$ & $0.24 \pm 0.53(-0.68$ to +2.15$)$ & $0.44 \pm 0.39(0.05$ to 2.15$)$ & $24.21 \pm 1.13$ & $19.53 \pm 3.40$ \\
\hline Sequential-phaco & 26 & $-0.95 \pm 1.26$ & $-0.09 \pm 0.51(-1.21$ to +1.12$)$ & $0.40 \pm 0.33(0.02$ to 1.21$)$ & $25.11 \pm 2.11$ & $17.92 \pm 5.19$ \\
\hline
\end{tabular}

Prediction error is the difference between actual and target refraction; $\mathrm{ME}=$ mean prediction error; Absolute prediction error is the absolute value of the prediction error; $\mathrm{MAE}=$ mean of the absolute prediction error; $\mathrm{AL}=$ axial length; $\mathrm{D}=$ diopter; $\mathrm{IOL}=$ intraocular lens; $\mathrm{SD}=$ standard deviation.

Using Levene's test, there was a statistically wider variance in predictive error in the phacovitrectomy group compared to the phaco-only control group ( $\mathrm{p}=$ 0.03 ) and a wider variance approaching significance between phacovitrectomy and sequential phaco $(\mathrm{p}=0.06)$.

Table 3 shows the proportion of eyes reaching pre-defined refractive targets. The phacovitrectomy group had a statistically lower proportion of eyes within $\pm 1.00 \mathrm{D}$ of target refraction compared to the phaco-only control group (78\% versus $95 \%, p=0.02)$. At other threshold levels, there was no statistically significant difference between the phacovitrectomy group and either of the 2 control groups, although the tendency was for phacovitrectomy to achieve lower rates to the controls at all thresholds.

\section{Discussion}

Our results have shown that OLCR-based optical biometry measurements with 
Table 3. Proportion of cases meeting refractive targets.

\begin{tabular}{|c|c|c|c|c|c|}
\hline \multirow{2}{*}{ Groups } & \multirow{2}{*}{ Cases $\mathrm{n}$} & \multicolumn{4}{|c|}{ Percentage of cases within target } \\
\hline & & $\pm 0.25 \mathrm{D}$ & $\pm 0.50 \mathrm{D}$ & $\pm 1.00 \mathrm{D}$ & $\pm 2.00 \mathrm{D}$ \\
\hline \multicolumn{6}{|l|}{ Phaco-vitrectomy } \\
\hline Macular hole & 18 & 17 & 56 & 72 & 100 \\
\hline$E R M$ & 18 & 33 & 72 & 83 & 94 \\
\hline Total cases & 36 & 25 & 64 & 78 & 97 \\
\hline Phaco-only & 41 & 41 & 71 & 95 & 98 \\
\hline Sequential Phaco & 26 & 38 & 73 & 92 & 100 \\
\hline
\end{tabular}

the Lenstar machine cause a myopic shift in phacovitrectomy outcomes (for ERM and MH) compared to standard cataract cases. The size of the myopic shift was approximately $-0.3 \mathrm{D}$ in our series. Myopic shift following phacovitrectomy for macular pathology has been previously documented in the context of ultrasound biometry [3]-[8] and more recently with the IOLMaster [10] [11], which is an optical biometer using PCI-based technology. Our results are significant in corroborating these earlier reports and in confirming that a similar myopic shift occurs in both PCI- and newer OLCR-based optical biometry platforms.

The myopic shift of $-0.3 \mathrm{D}$ with the Lenstar in our series was in close agreement with 2 prior studies using the IOLMaster. Both Falkner-Radler et al. [10] and Kim et al. [11] found a myopic shift of $-0.4 \mathrm{D}$ when comparing combined phacovitrectomy for macular pathology with routine cataract surgery, using the IOLMaster with the SRK/T formula. Not all studies with the IOLMaster, however, have reported a myopic shift. Manvikar et al. found no shift with combined phacovitrectomy for ERM's and macular holes versus standard cataract surgery [9]. They used the IOLMaster with the Haigis formula. Use of the Haigis formula per se is unlikely to have influenced their outcomes, as the authors reported no statistical difference in absolute predictive error between the SRK/T formula and the Haigis formula in either arm of their series. Interestingly, they performed primary posterior capsulotomy with a vitrectomy cutter during phacovitrectomy. This was not undertaken in our series or in those by Falkner-Radler et al. [10] or Kim et al. [11]. Whether capsulotomy negated the tendency towards myopia in their series is difficult to ascertain as there are no reports on the refractive influence of this maneuver.

The myopic shift observed with both ultrasound and optical biometry after phacovitrectomy is likely multifactorial and there have been many hypothesized causes. Removal of the vitreous gel may alter the ocular refractive index [12] and this has been calculated to contribute from $-0.13 \mathrm{D}$ to $-0.5 \mathrm{D}$ in myopic shift [6] [13] [14]. Vitrectomy may also influence the effective lens position and hence refractive outcome. Greater IOL movement has been observed after phacovitrectomy compared to standard cataract surgery [10]. Intravitreal gas tamponade may be a contributor to IOL mobility and hence myopic shift. Schweitzer et al. observed a myopic shift of $-0.5 \mathrm{D}$ in phacovitrectomy with gas tamponade relative to phacovitrectomy without gas tamponade [15] although other studies have 
not observed this association [8] [9]. In our own series there was no correlation between gas tamponade and increasing myopic shift on multivariate analysis.

Perhaps the prime suspect in myopic shift with phacovitrectomy is an inaccuracy or bias in axial length $(\mathrm{AL})$ readings. Errors in $\mathrm{AL}$ have been estimated to contribute $54 \%$ of the overall prediction error in routine cataract cases [16]. In the context of macular pathology this effect may be greater.

Ultrasound biometry measures AL by reflecting sound waves off the internal limiting membrane [17]. The focusing plane of the retina, however, corresponds to the outer limiting membrane in close proximity to the retinal pigment epithelium (RPE). Ultrasound biometers therefore make assumptions of retinal thickness in measuring AL. In a thickened retina, the ultrasound presumes normal thickness and may underestimate AL, creating a myopic shift. Longitudinal studies have confirmed changes in OCT macula thickness over time cause changes in ultrasound AL measurement [4].

Both PCI- and OLCR-based optical biometry machines, on the other hand, use the principle of laser interferometry whereby infra-red laser reflected off the $\mathrm{RPE}$ is used to measure AL. The two technologies differ firstly in the light source used (with PCI using a $780 \mathrm{~nm}$ multimode diode while OLCR uses an $820 \mathrm{~nm}$ superluminescent diode) and secondly in the way the interferometer interprets signals [18]. Comparisons between PCI (IOLMaster) and OLCR (Lenstar) biometry have shown highly agreeable $\mathrm{AL}$ readings although distinct biometric constants are still recommended [19] [20] [21]. Since optical biometry relies on reflection off the RPE, these techniques should be less affected by increased macular thickness and inner retinal disturbances compared to ultrasound. The myopic shift seen with both the Lenstar and the IOLMaster (and which is comparable in size to the shift in ultrasound [11]) is therefore all the more intriguing. Manvikar et al. have suggested that in the presence of macula pathology, the reflecting surface for optical biometers may no longer be the RPE [9]. Specular reflections, for example, may occur off epiretinal membranes or other anatomical aberrations. However, one might expect such a phenomenon to produce markedly abnormal scans with altered peaks and reduced signal-to-noise ratio, which has not been clinically observed. Alternatively, perhaps the similarity in size of myopic shift across different biometry platforms suggests the majority of myopic shift emanates not from biometry errors per se but from factors inherent to vitrectomy surgery (such as changes in refractive index and IOL positioning as discussed earlier). Interestingly, the sequential-phaco control group in our study demonstrated an almost identical myopic shift $(-0.3 \mathrm{D})$ to the phacovitrectomy group. This again supports the notion that factors inherent to vitrectomy surgery (or a history of vitrectomy surgery) play a role in myopic shift quite apart from biometry errors related to active macular disease. Other groups have reported a similar myopic shift after sequential surgery [22]. A myopic shift has also been observed after pseudophakic vitrectomy for a variety of indications, which again suggests a role for factors inherent to vitrectomy surgery as opposed to biometry errors [23] [24]. 
Besides a shift to myopia, phacovitrectomy produced more variable refractive outcomes in our series compared to routine cataract surgery with a statistically larger variance in predictive error $(\mathrm{p}=0.03)$. A similar observation was made by Falkner-Radler et al. [10]. Moreover, a lower proportion of phacovitrectomy cases achieved $\pm 1.00 \mathrm{D}$ of target refraction compared to phaco-only cases ( $\mathrm{p}=$ 0.02 ). It is important to emphasize that an acceptable proportion of phacovitrectomy cases in our series still achieved refractive targets. In the United Kingdom, national guidelines on cataract surgery issued by the Royal College of Ophthalmologists suggest aiming for $55 \%$ of cases within $\pm 0.50 \mathrm{D}$ (a target our phacovitrectomy group surpassed at $64 \%$ ) and $85 \%$ of cases within $\pm 1.00 \mathrm{D}$ (a target our phacovitrectomy group approached at 78\%) [25]. Nevertheless, in light of our results, we believe patients undergoing phacovitrectomy should be counselled that refractive outcomes can be more variable by comparison to standard cataract surgery.

In the debate regarding combined versus sequential surgery for macular pathology, we could find no convincing evidence in a head-to-head statistical comparison that combined was less reliable than sequential. The variance in the prediction error, the proportion of cases achieving refractive targets (Table 2) and the MAE (Table 2) between the 2 groups all tended to favor sequential compared to combined, but not at a statistically significant level ( $p>0.05)$. This may represent a lack of statistical power in our study as the sequential-phaco group was smallest $(n=26)$. We recommend further studies with larger recruitment numbers to better assess the reliability of combined versus sequential surgery. Should a discrepancy be found, we suggest its clinical effect is likely to be small. For the cohort of patients with sub-optimal visual potential due to macular disease, the time-delay and additive surgical risks associated with a sequential procedure are likely to hold greater sway in their decision than any proposed benefit in the statistical variance of refractive outcomes.

\section{Conclusion}

our study confirms that OLCR-based optical biometry produces a myopic shift in both phacovitrectomy and sequential cataract surgery (after prior vitrectomy) compared to routine cataract surgery cases. For surgeons seeking to optimize their refractive outcomes in these scenarios, we recommend regularly auditing outcomes. The use of customized biometric constants in these scenarios, distinct from routine cataract surgery should help to correct for myopic shift. Refractive outcomes in our series were statistically more variable in phacovitrectomy than that with routine cataract surgery. Nevertheless, refractive outcomes with phacovitrectomy were comparable with nationally agreed targets for cataract surgery in the United Kingdom, confirming that phacovitrectomy remains a viable approach for patients with macula pathology. No statistically conclusive evidence was found that phacovitrectomy is less reliable than sequential cataract surgery but more studies are suggested to fully elucidate any discrepancies. 


\section{References}

[1] Steel, D.H.W. (2007) Phacovitrectomy: Expanding Indications. Journal of Cataract \& Refractive Surgery, 33, 933-936.

[2] Muselier, A., Dugas, B., Burelle, X., Passemard, M., Hubert, I., Mathieu, B., Berrod, J.P., Bron, A.M. and Creuzot-Garcher, C. (2010) Macular Hole Surgery and Cataract Extraction: Combined vs. Consecutive Surgery. American Journal of Ophthalmology, 150, 387-391.

[3] Patel, D., Rahman, R. and Kumarasamy, M. (2007) Accuracy of Intraocular Lens Power Estimation in Eyes Having Phacovitrectomy for Macular Holes. Journal of Cataract \& Refractive Surgery, 33, 1760-1762.

[4] Kovács, I., Ferencz, M., Nemes, J., Somfai, G., Salacz, G. and Récsán, Z. (2007) Intraocular Lens Power Calculation for Combined Cataract Surgery, Vitrectomy and Peeling of Epiretinal Membranes for Macular Oedema. Acta Ophthalmologica Scandinavica, 85, 89-91. https://doi.org/10.1111/j.1600-0420.2006.00772.x

[5] Suzuki, Y., Sakuraba, T., Mizutani, H., Matsuhashi, H. and Nakazawa, M. (2001) Postoperative Complications after Simultaneous Vitrectomy and Cataract Surgery. Ophthalmic Surgery Lasers and Imaging, 32, 391-396.

[6] Shioya, M., Ogino, N. and Shinjo, U. (1997) Change in Postoperative Refractive Error When Vitrectomy Is Added to Intraocular Lens Implantation. Journal of Cataract \& Refractive Surgery, 23, 1217-1220.

[7] Iwase, T. and Sugiyama, K. (2006) Investigation of the Stability of One-Piece Acrylic Intraocular Lenses in Cataract Surgery and in Combined Vitrectomy Surgery. British Journal of Ophthalmology, 90, 1519-1523.

[8] Jeoung, J.W., Chung, H. and Yu, H.G. (2007) Factors Influencing Refractive Outcomes after Combined Phacoemulsification and Pars Plana Vitrectomy: Results of a Prospective Study. Journal of Cataract \& Refractive Surgery, 33, 108-114.

[9] Manvikar, S.R., Allen, D. and Steel, D.H.W. (2009) Optical Biometry in Combined Phacovitrectomy. Journal of Cataract \& Refractive Surgery, 35, 64-69.

[10] Falkner-Radler, C.I., Benesch, T. and Binder, S. (2008) Accuracy of Preoperative Biometry in Vitrectomy Combined with Cataract Surgery for Patients with Epiretinal Membranes and Macular Holes. Results of a Prospective Controlled Clinical Trial. Journal of Cataract \& Refractive Surgery, 34, 1754-1760.

[11] Kim, M., Kim, H.E., Lee, D.H., Koh, H.J., Lee, S.C. and Kim, S.S. (2015) Intraocular Lens Power Estimation in Combined Phacoemulsification and Pars Plana Vitrectomy in Eyes with Epiretinal Membranes: A Case-Control Study. Yonsei Medical Journal, 56, 805. https://doi.org/10.3349/ymj.2015.56.3.805

[12] Sardar, D.K., Swanland, G.-Y., Yow, R.M., Thomas, R.J. and Tsin, A.T.C. (2007) Optical Properties of Ocular Tissues in the near Infrared Region. Lasers in Medical Science, 22, 46-52. https://doi.org/10.1007/s10103-006-0421-y

[13] Mehdizadeh, M. and Nowroozzadeh, M.H. (2009) Postoperative Induced Myopia in Patients with Combined Vitrectomy and Cataract Surgery. Journal of Cataract \& Refractive Surgery, 35, 798-799.

[14] Hamoudi, H. and La Cour, M. (2013) Refractive Changes after Vitrectomy and Phacovitrectomy for Macular Hole and Epiretinal Membrane. Journal of Cataract \& Refractive Surgery, 39, 942-947.

[15] Schweitzer, K.D. and García, R. (2008) Myopic Shift after Combined Phacoemulsification and Vitrectomy with Gas Tamponade. Canadian Journal of Ophthalmology, 43, 581-583. https://doi.org/10.3129/i08-135

[16] Olsen, T. (1992) Sources of Error in Intraocular Lens Power Calculation. Journal of 
Cataract \& Refractive Surgery, 18, 125-129.

[17] Olsen, T. (2007) Calculation of Intraocular Lens Power: A Review. Acta Ophthalmologica Scandinavica, 85, 472-485. https://doi.org/10.1111/j.1755-3768.2007.00879.x

[18] Goldblum, D. (2015) Physics Lesson : Differences in PCI and OLCR Optical Biometry. Cataract \& Refractive Surgery Today, 41-43.

[19] Rohrer, K., Frueh, B.E., Wälti, R., Clemetson, I.A., Tappeiner, C. and Goldblum, D. (2009) Comparison and Evaluation of Ocular Biometry Using a New Noncontact Optical Low-Coherence Reflectometer. Ophthalmology, 116, 2087-2092.

[20] Cruysberg, L.P.J., Doors, M., Verbakel, F., Berendschot, T.T.J.M., De Brabander, J. and Nuijts, R.M.M. (2010) Evaluation of the Lenstar LS 900 Non-Contact Biometer. British Journal of Ophthalmology, 94, 106-110. https://doi.org/10.1136/bjo.2009.161729

[21] Buckhurst, P.J., Wolffsohn, J.S., Shah, S., Naroo, S.A., Davies, L.N. and Berrow, E.J. (2009) A New Optical Low Coherence Reflectometry Device for Ocular Biometry in Cataract Patients. British Journal of Ophthalmology, 93, 949-953. https://doi.org/10.1136/bjo.2008.156554

[22] Bilińska, E., Nawrocki, J., Suprunowicz, I. and Omulecki, W. (2002) Refraction Changes after Cataract Extraction with IOL Implantation in the Eyes with Previous Performed Vitrectomy. Klinika Oczna, 104, 344-346.

[23] Hamoudi, H., Kofod, M. and La Cour, M. (2013) Refractive Change after Vitrectomy for Epiretinal Membrane in Pseudophakic Eyes. Acta Ophthalmologica, 91, 434436. https://doi.org/10.1111/j.1755-3768.2012.02574.x

[24] Byrne, S., Ng, J., Hildreth, A., Danjoux, J.-P. and Steel, D.H. (2008) Refractive Change Following Pseudophakic Vitrectomy. BMC Ophthalmology, 8, 19. https://doi.org/10.1186/1471-2415-8-19

[25] Royal College of Ophthalmologists (2010) Cataract Surgery Guidelines. https://www.rcophth.ac.uk/standards-publications-research/clinical-guidelines

\section{Scientific Research Publishing}

Submit or recommend next manuscript to SCIRP and we will provide best service for you:

Accepting pre-submission inquiries through Email, Facebook, LinkedIn, Twitter, etc. A wide selection of journals (inclusive of 9 subjects, more than 200 journals)

Providing 24-hour high-quality service

User-friendly online submission system

Fair and swift peer-review system

Efficient typesetting and proofreading procedure

Display of the result of downloads and visits, as well as the number of cited articles

Maximum dissemination of your research work

Submit your manuscript at: http://papersubmission.scirp.org/

Or contact ojoph@scirp.org 